\title{
Identification of Key Genes Between Lung Adenocarcinoma and Lung Squamous Cell Carcinoma by Bioinformatics Analysis
}

\author{
Jinru Xue, MS ${ }^{a}$, Yu Yang, MS ${ }^{b}$, Bo Jiang, MD ${ }^{b}$, Linxue Qian, MD ${ }^{b}$, Xian-Quan Shi PhD ${ }^{b, *}$ \\ ${ }^{a}$ Department of Thoracic surgery, China-Japan Union Hospital of Jilin University, Changchun, Jilin, China $;{ }^{b}$ Department of Ultrasound, \\ Beijing Friendship Hospital, Capital Medical University, Beijing, China
}

Received March 03, 2020; revision received March 20, 2020; accepted May 20, 2020

Objective: To explore differentially expressed genes (DEGs) between lung adenocarcinoma (LUAD) and lung squamous cell carcinoma (LUSC).

Methods: Based on GEO database, we used R software to identify the DEGs and conducted the bioinformatics analysis to explore the molecular mechanisms of DEGs and constructed PPI network to find the key DEGs. Then we assessed the effect of the eligible key DEGs on survival in LUAD by Kaplan-Meier plotter online tool.

Results: GSE10245 was downloaded from the GEO database, which contained a total of 58 tissue samples, including 18 LUSC and 40 LUAD. We identified 784 DEGs between LUAD and LUSC. DEGs were enriched in statistical significant GO annotation 201 items and KEGG pathways 17 items. By constructed PPI network, we obtained 10 hub genes. Of which, five genes were significantly correlated with the overall survival of LUAD.

Conclusions: P2RY1, CHRM3, LPAR3, NMU, and S1PR5 may be the potential prognostic markers and therapeutic targets for LUAD.

Keywords: Bioinformatics analysis; LUAD; Differentially expressed genes

Advanced Ultrasound in Diagnosis and Therapy 2020;04:335-342

DOI: $10.37015 / A U D T .2020 .200011$

$\mathrm{N}$ on-small cell lung cancer (NSCLC) accounts for approximately $85 \%$ of LC [1], which the morbidity and mortality rank first in malignant tumors [2]. The incidence rates of lung adenocarcinoma (LUAD, approximately $50 \%$ of cases) and lung squamous cell carcinoma (LUSC, approximately $30 \%$ of cases) comprises $70 \%$ of NSCLC, which are the predominant histological subtypes of NSCLC [2-4]. Until recently, surgery is the recommended treatment for stage I - II NSCLC patients, 5-year survival of clinical stage I NSCLC and pathological stage I NSCLC after surgical resection can reach $68-92 \%$ and $73-90 \%$, respectively $[5,6]$. However, due to lack of early effective diagnostic methods, $70 \%$ of LC patients are diagnosed at advantage stage when the tumor lose the opportunity of surgical resection [7]. Experimental chemotherapy is the main treatment method for these patients, though the clinically outcomes are poor.

Attributed to the development of next-generation sequencing, the treatment strategies for advanced NSCLC patients have evolved to effective regimens that are targeted to specific molecular subtypes and specific genomic abnormalities of tumors [8,9]. According to statistics, up to $69 \%$ of advanced NSCLC patients have a potentially actionable molecular target [10]. Based on cell origin and different growth patterns, the specific

* Corresponding author: Department of Ultrasound, Beijing Friendship Hospital, Capital Medical University, 95 Yongan Road, Beijing, China

e-mail: sonoshixq@ccmu.edu.cn unrestricted use, distribution and reproduction in any medium provided that the original work is properly attributed. 
targeted treatment produces modest improvements in survival for NSCLC patients [11-16]. However, Targeted treatment is limited to certain subsets of NSCLC patients, and patients with different histological subtypes and molecular abnormalities have different response to the same targeted therapy medications. For example, epidermal growth factor receptor (EGFR)-tyrosine kinase inhibitors (TKIs) had great curative effect for patients with EGFR-mutated LUAD, but almost ineffective in EGFR-mutated LUSC [15-17]. Therefore, further progress is expected to discovery new and potentially great genotype-directed therapy targets for more subgroup's patients with NSCLC, which could be made a notable impact in outcomes for advanced NSCLC patients.

Since gene microarray and bioinformatics methods are broadly used in cancer researches, further identify the pathogenesis and search for potential specific diagnostic, prognostic markers and therapeutic targets have become possible. As a result, it is important to clarify the differentially expressed genes (DEGs), biological processes and molecular mechanisms of LUAD and LUSC from the perspective of biomedicine. In this study, we explored DEGs by comparison the genes expression profiles of tumor tissues between LUSC and LUAD patients from Gene Expression Omnibus (GEO) database (GEO database). The DEGs were conducted gene ontology (GO) and Kyoto Encyclopedia of Genes and Genomes (KEGG) pathway analysis. Furthermore, Protein-protein interaction (PPI) network, module analysis, and survival analysis were performed.

\section{Materials and methods}

\section{Microarray data}

GSE10245 was downloaded from the GEO DataSets, annotated by Human Genome U133 Plus 2.0 (Affymetrix, USA), and the chip platform was GPL570. A total of 58 tissue samples were collected, including 40 LUAD and 18 LUSC.

\section{Data analysis and identification of DEGs}

Software R x64 (version 3.4.3) was used to analyze the GSE10245 expression profile dataset, identify the DEGs compared LUAD with LUSC, plot heatmap and volcanic map. The DEGs screening criteria in this study were adjusted $P$ value $<0.05$ and $|\log \mathrm{FC}|>1$.

\section{GO and KEGG analysis of DEGs}

GO and KEGG enrichment analysis were applied by Visualization and Integrated Discovery (DAVID, https:// david.ncifcrf.gov/) program. $P<0.05$ was regarded statistically significant.

\section{PPI network establishment and modules selection}

STRING database (https://string-db.org/) was used to infer the PPI information. Entering all of DEGs names in STRING for a search, the combined score $>0.9$ was considered significant. Molecular Complex Detection (MCODE), a plus in Cytoscape version 3.6.1, was used to screen the modules from PPI network. Degree Cutoff $=3$, Node Score Cutoff $=0.2$, K-Core $=2$, Max.Depth $=100$ were set as the cut-off criterion. Modules with MCODE score $>8$ were presented. Eligible hub genes were selected as potential key genes.

\section{Survival analysis}

The Kaplan-Meier plotter online tool (http://kmplot. $\mathrm{com} /$ ) was applied to assess the effect of the eligible hub genes on survival[18]. The Logrank test $P<0.05$ was statistically significant.

\section{Results}

\section{Identification of DEGs}

A total of 784 DEGs were identified, 517 genes were upregulated and the remaining 267 genes were downregulated. Then we plotted heatmap of the top 100 DEGs (top 50 upregulated and downregulated genes), as discribed in Figure 1. All of the 20460 genes were represented on the volcano map, as presented in Figure 2. Red dots represented 784 DEGs and black dots represented the remaining genes with no different expression.

\section{GO and KEGG analysis of DEGs}

All the DEGs names were submitted to DAVID (https://david.ncifcrf.gov/) for GO and KEGG analysis, and we found statistically significant GO analysis 201 items and KEGG pathways 17 items. For the biological process (BP), DEGs were significantly aggregated in epidermis development, keratinocyte differentiation, negative regulation of endopeptidase activity, establishment of skin barrier, keratinization and peptide cross-linking, as shown in table 1. For the cellular component (CC), DEGs were significantly assembled in the extracellular space, cornified envelope, extracellular exosome, extracellular region, apical plasma membrane and apicolateral plasma membrane, as shown in table 1. For the molecular function (MF), DEGs were markedly enriched in serine type endopeptidase inhibitor activity and structural molecule activity, as shown in table 1. KEGG analysis showed that DEGs were significantly enriched in cell cycle, as shown in table 1.

\section{PPI network construction and modules selection}

All DEGs names were submitted to STRING and 
PPI network was constructed. As shown in Figure 3, 344 nodes and 562 edges were identified, PPI enrichment $\mathrm{p}$ value $<1.0 \mathrm{e}-16$. Used Cytoscape plugin to identify the hub gene modules, and the cut-off criterion was set as degrees $>8$. Ten genes constituted one significant module, they were $\mathrm{PMCH}$ (pro-melanin concentrating hormone), NTS (neurotensin), P2RY1 (purinergic receptor P2RY1), CHRM3 (cholinergic receptor muscarinic 3), NMU (neuromedin U), AGT (angiotensinogen), CXCL6 (C-X-C motif chemokine ligand 6), LPAR3 (lysophosphatidic acid receptor 3), HCAR3 (hydroxycarboxylic acid receptor 3) and S1PR5 (sphingosine-1-phosphate receptor 5), as shown in Figure 4. These ten hub genes were considered to be the major DEGs between LUAD and LUSC.

Table 1 GO and KEGG pathways analysis of differentially expressed genes (DEGs) with $P<0.05$

\begin{tabular}{|c|c|c|c|c|c|}
\hline Category & Term & Count & $\%$ & $P$ Value & FDR \\
\hline GOTERM_BP_DIRECT & GO:0008544 epidermis development & 25 & 3.27654 & $8.44 \mathrm{E}-15$ & $1.51 \mathrm{E}-11$ \\
\hline GOTERM_BP_DIRECT & GO:0030216 keratinocyte differentiation & 22 & 2.883355 & $6.51 \mathrm{E}-13$ & $1.16 \mathrm{E}-09$ \\
\hline GOTERM_BP_DIRECT & GO:0010951 negative regulation of endopeptidase activity & 19 & 2.49017 & $9.47 \mathrm{E}-07$ & 0.00169 \\
\hline GOTERM_BP_DIRECT & GO:0061436 establishment of skin barrier & 8 & 1.048493 & $2.79 \mathrm{E}-06$ & 0.004984 \\
\hline GOTERM_BP_DIRECT & GO:0031424 keratinization & 11 & 1.441678 & $1.23 \mathrm{E}-05$ & 0.022017 \\
\hline GOTERM_BP_DIRECT & GO:0018149 peptide cross-linking & 11 & 1.441678 & $1.81 \mathrm{E}-05$ & 0.032242 \\
\hline GOTERM_CC_DIRECT & GO:0005615 extracellular space & 100 & 13.10616 & 7.97E-11 & $1.10 \mathrm{E}-07$ \\
\hline GOTERM_CC_DIRECT & GO:0001533 cornified envelope & 14 & 1.834862 & $8.95 \mathrm{E}-09$ & $1.24 \mathrm{E}-05$ \\
\hline GOTERM_CC_DIRECT & GO:0070062 extracellular exosome & 162 & 21.23198 & $1.35 \mathrm{E}-08$ & $1.88 \mathrm{E}-05$ \\
\hline GOTERM_CC_DIRECT & GO:0005576 extracellular region & 100 & 13.10616 & $6.99 \mathrm{E}-07$ & $9.69 \mathrm{E}-04$ \\
\hline GOTERM_CC_DIRECT & GO:0016324 apical plasma membrane & 30 & 3.931848 & $1.96 \mathrm{E}-06$ & 0.002714 \\
\hline GOTERM_CC_DIRECT & GO:0016327 apicolateral plasma membrane & 7 & 0.917431 & $2.43 \mathrm{E}-05$ & 0.033725 \\
\hline GOTERM_MF_DIRECT & GO:0005198 structural molecule activity & 32 & 4.193971 & 4.72E-09 & $7.20 \mathrm{E}-06$ \\
\hline GOTERM_MF_DIRECT & GO:0004867 serine-type endopeptidase inhibitor activity & 17 & 2.228047 & $6.75 \mathrm{E}-07$ & 0.00103 \\
\hline KEGG_PATHWAY & hsa04110:Cell cycle & 18 & 2.359108781 & 4.11E-06 & 0.005266549 \\
\hline
\end{tabular}

BP, Biological process; CC, Cellular component; MF, Molecular function; GO, Gene ontology; KEGG, Kyoto encyclopedia of genes and genomes; FDR, false discovery rate. E represents base of 10 to the power.

\section{Survival analysis}

Ten hub genes underwent survival analysis using Kaplan-Merier online tool (http://kmplot.com/analysis/), including 675 LUSC and 866 LUAD). The results showed that the LUAD patients with high expression of P2RY1 (HR=0.7 (0.55-0.89), logrank $P=0.0031)$, CHRM3 (HR $=0.72(0.56-0.92)$, logrank $P=0.0085)$ and LPAR3 (HR=0.54 (0.42-0.69), logrank $P=6.8 \mathrm{e}-$ 07 ) had significantly longer overall survival time than those with low expression groups, as shown in Figure 5. And the LUAD patients with low expression of NMU (HR=1.69(1.33-2.14), logrank $P=1.3 \mathrm{e}-05)$ and S1PR5 (HR=1.93(1.52-2.45), logrank $P=5.2 \mathrm{e}-08)$ had significantly longer overall survival time than those with high expression group, as shown in Figure 5.

\section{Discussion}

NSCLC has particularly high intratumoral heterogeneity due to distinct molecular features. Understanding the molecular mechanisms of NSCLC is critical for diagnosis, treatment and prognosis. In this study, we identified 784 DEGs either upregulated or downregulated in all compared LUAD with LUSC. Function annotation revealed statistically significant GO analysis 201 items and KEGG pathways 17 items. Through constructing PPI network, ten hub genes were considered to be the key DEGs. Three downregulated (P2RY1, CHRM3, and LPAR3) genes and two upregulated (NMU and S1PR5) genes were significantly related to worse overall survival in LUAD patients. 


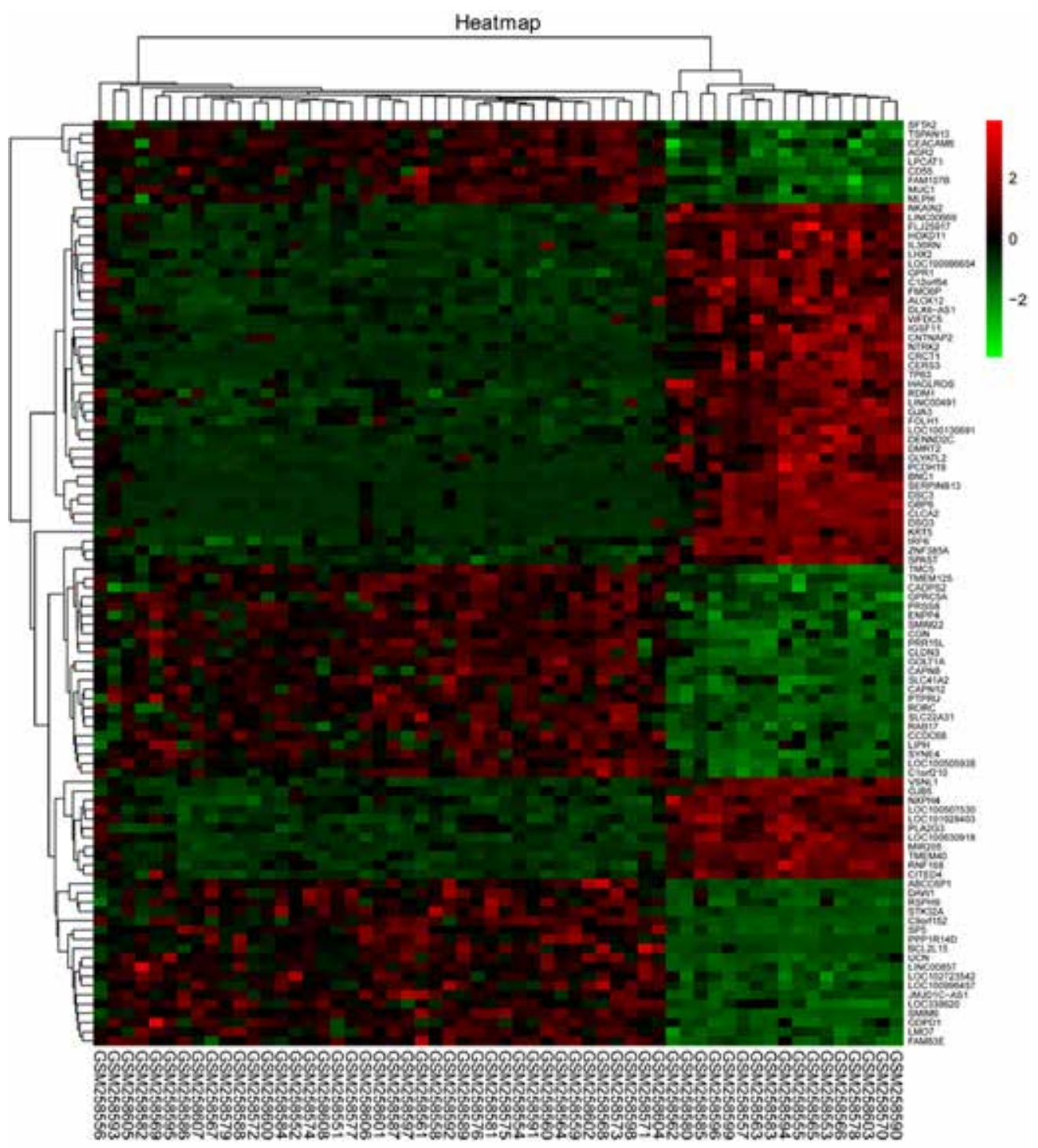

Figure 1 Heatmap of top 50 upregulated and downregulated genes differentially expressed genes (DEGs), red ones represented upregulation and green represented downregulation.

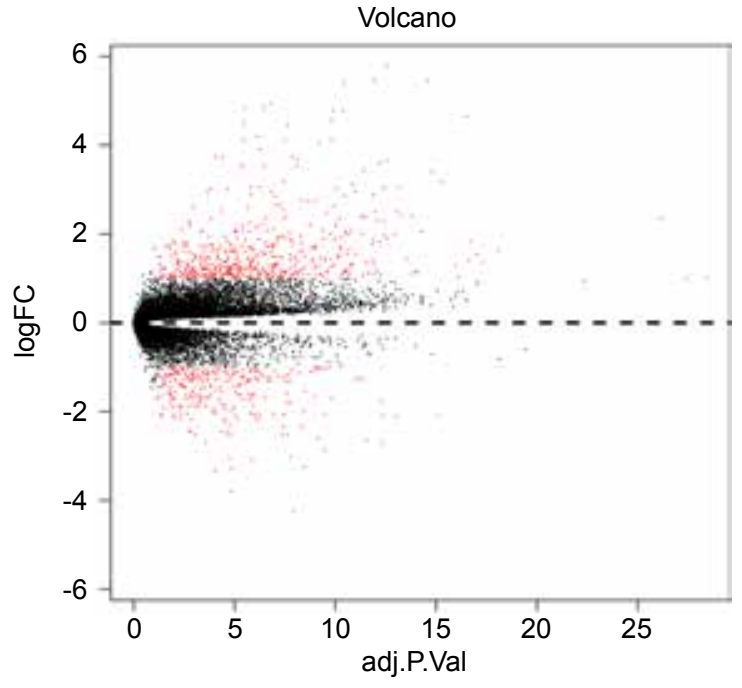

Figure 2 Volcano plot of the 20460 genes. Red dots represented the 784 differentially expressed genes (DEGs) and black dots represented the remaining genes with no different expression.
For cellular component (CP), DEGs were markedly located in the extracellular exosomes, extracellular space, cornified envelope, and extracellular region, as shown in table 1. Exosomes, which contain proteins, nucleic acids, lipids and the encapsulated contents, play an vital role in cell communication, tumor progression, drug resistance, and recent years have also play an important role in liquid biopsy of NSCLC [19-21]. It provides a new basis for early diagnosis, precision medicine and prognosis of NSCLC. However, the study of exosomes still is in their infancy, whether it can be applied in clinic remains need to be confirmed by further research. For molecular function (MF), DEGs were mainly promoted serine type endopeptidase inhibitor activity and structural molecule activity. Serine type endopeptidase inhibitor is a subtype of serine protease inhibitor, which is termed serpins. Serpins sit at intersections of normal physiology and pathology, both ensuring normal biological function 


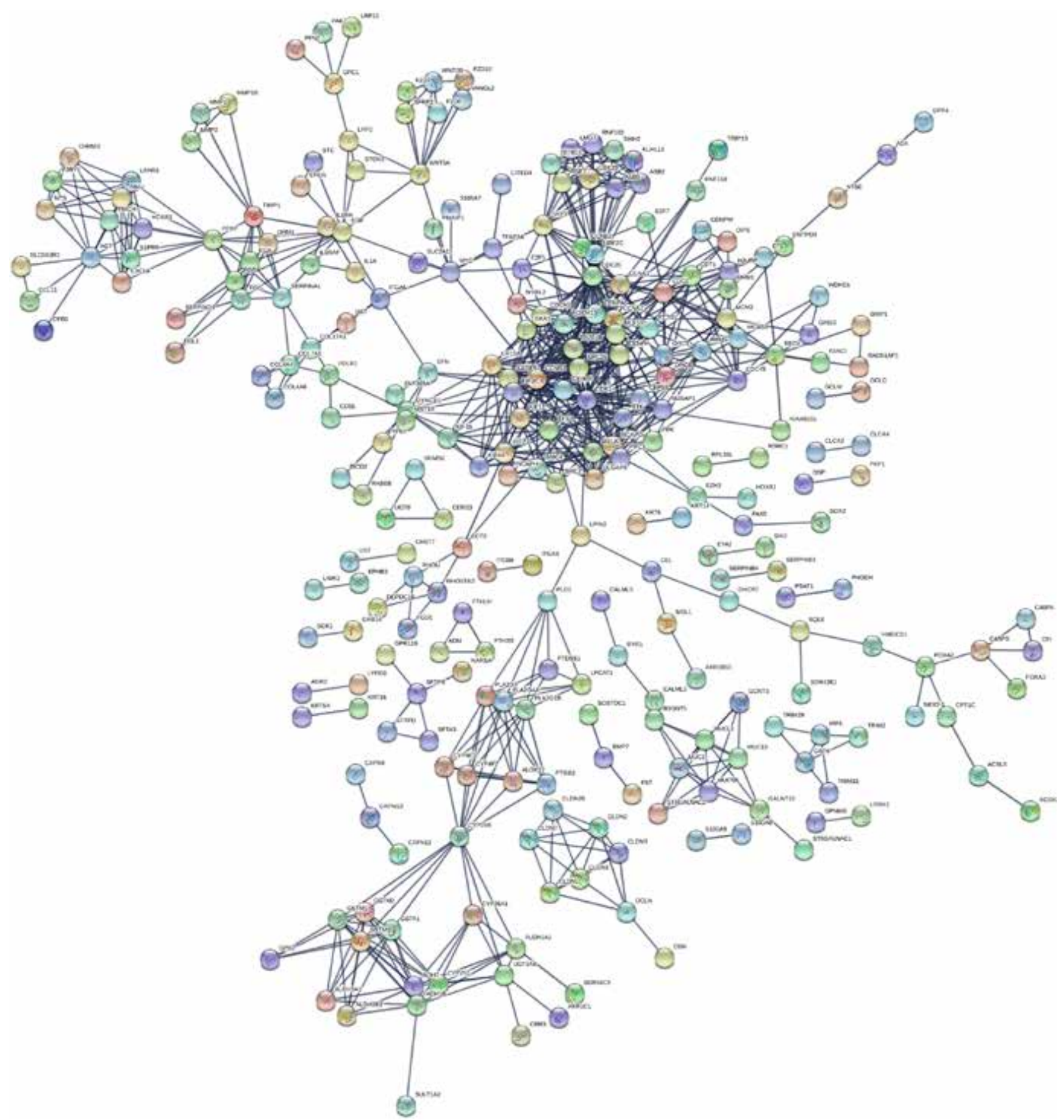

Figure 3 Protein-protein interaction (PPI) network of the 784 differentially expressed genes (DEGs).

but also, when dysfunctional, leading to disease [22-25]. It is involved in a variety of biological processes, such as inflammation, immune response, cell invasion, tissue remodeling and cancer development [22,26-28]. With cancers, altered levels of serpins and related proteases are correlated with invasion and metastasis, even can serve as markers for aggression [29]. For biological process (BP) enrichment, DEGs were mainly involved in epidermis development, keratinocyte differentiation, keratinization and peptide cross-linking. It mainly refers to the process of epidermal cells from formation to maturity over time. In vivo, epithelial cells and mesenchymal cells can be converted under certain conditions during the epithelia cells development. The epithelial-mesenchymal transition (EMT) is critical for cancer progression [3033]. EMT is driven by transcriptional factors, results in enhancer migration and invasive potential of epithelial cells, and is critical for the metastatic spread of epithelial tumors $[30,34,35]$. 


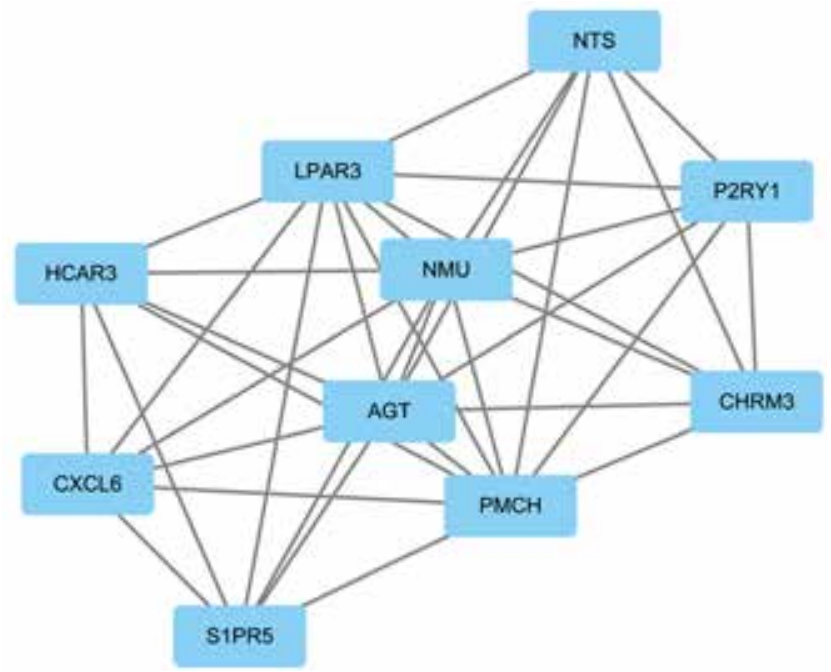

Figure 4 Significant module inferred from protein-protein interaction network. gene, so its role in NSCLC is less studied. S1PR5 is a constitutively $\mathrm{G}$ protein-coupled receptor with the ability to inhibit cell proliferation and induce cell migration in cancer by $\mathrm{G} \alpha 12 / 13$-evoked stimulation of Rho, suggesting an inhibitory role of S1PR5 [52-54]. S1PR5 has also been shown to have pro-survival effect by inducing autophagy and S1PR5 activation was found to be involved by applying small interfering RNA and dihydro-S1P in cancer $[55,56]$.

\section{Conclusion}

In conclusion, our study discovered a series of key targets for further research on molecular mechanisms of LUAD by a comprehensive bioinformatics analysis, which provides new therapeutic and prognostic clues for LUAD patients, Other than bioinformatics exploration,
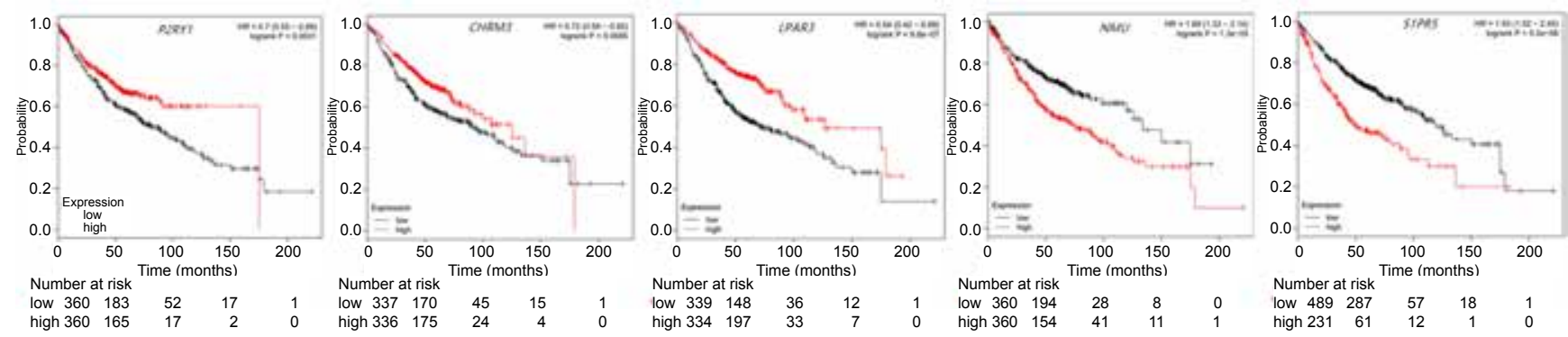

Figure 5 Prognostic values of the five key DEGs in LUAD patients.

P2RY1 encodes a G-protein couples receptors, adenine di-phosphate (ADP) is a physiological agonist for P2RY1 [36]. P2RY1 couples to phospholipase $\mathrm{C}$, which triggers $\mathrm{Ca}^{2+}$ release from the intracellular reservoir, leading to reversible platelet aggregation [37]. The P2RY1 polymorphism might participate in the control of the various physiological functions, and negative regulation of P2RY1 in the multidrug chemoresistance of bladder cancer cells was reported $[38,39]$. The copy number variation $(\mathrm{CNV})$ frequencies for pharmacogenes using The Cancer Genome Atlas dataset showed that P2RY1 may play a role in determining individual variations in drug responses in NSCLC patients [40]. CHRM3 is one of the muscarinic receptors and plays an important role in many kinds of cancer, such as breast cancer, colon cancer, and lung cancer [41]. Experienced study revealed that CHRM3 highly expressed in NSCLC and enhanced the expression and activity of matrix metalloproteinase 9 (MMP9) through $\mathrm{PI} 3 \mathrm{~K} / \mathrm{Akt}$, which regulated the transcription of MMPs and EMT-related genes in NSCLC $[42,43]$. It has demonstrated overexpression of CHRM3 in endometrial carcinoma patients, as an prognostic factor, had a shorter overall survival time [44]. NMU encodes a member of the neuromedin family of neuropeptides and is associated with cell motility, invasion, and resistance to anoikis [45]. In NSCLC pathogenesis, NMU might be a target of the lung metastasis suppressor effect of RhoGD12 and RhoGD12 reconstitution in NSCLC cells without expression resulted in lung metastasis suppression [46]. In Shuangjie You's study, it showed that NMU was the candidate promoting the tumorigenesis and alectinib resistance in NSCLC [47]. Sweta Rani et al reported that NMU was a candidate prognostic indicator and therapeutic target for HER2- overexpressing tumors, which associated with poor outcomes and effected sensitivity to lapatinib, trastuzumab, neratinib and afatinib in patients with HER2 overexpressing tumors [45]. LPAR3 indicates several biological functions, such as cell proliferation, migration, and tumor progression [48]. LPAR3 abnormally expressed in many cancer cells, such as ovarian cancer, colorectal cancer, and LC [49-51]. The developmental study found that aberrant expression of LPAR3 due to aberrant DNA methylation induced inhibition of lung cancer cell migration in rat, which suggested that LPAR3 may be promising chemotherapeutic target gene and chemoprevention for LC [51]. S1PR5 is the most recently deorphanized 
functional researches that further delineate the effects of the key DEGs are needed to validate their specific roles in the pathogenesis of LUAD, even and in the diagnosis, individualized treatment, and prognosis of LUAD.

\section{Conflict of Interest}

The authors declare no conflict of interests.

\section{References}

[1] Travis WD, Brambilla E, Nicholson AG, Yatabe Y, Austin JHM, Beasley MB, et al The 2015 world health organization classification of lung tumors: impact of genetic, clinical and radiologic advances since the 2004 classification. $J$ Thorac Oncol 2015;10:1243-1260.

[2] Bray F, Ferlay J, Soerjomataram I, Siegel RL, Torre LA, Jemal A. Global cancer statistics 2018: GLOBOCAN estimates of incidence and mortality worldwide for 36 cancers in 185 countries. CA Cancer J Clin 2018;68:394-424.

[3] Siegel RL, Miller KD, Jemal A. Cancer statistics, 2017. CA Cancer J Clin 2017;67:7-30.

[4] Travis WD, Brambilla E, Nicholson AG, Yatabe Y, Austin JHM, Beasley MB, et al. The 2015 world health organization classification of lung tumors: impact of genetic, clinical and radiologic advances since the 2004 classification. $J$ Thorac Oncol 2015;10:1243-1260.

[5] Goldstraw P, Chansky K, Crowley J, Rami-Porta R, Asamura $\mathrm{H}$, Eberhardt WE, et al. The IASLC lung cancer staging project: proposals for revision of the TNM stage groupings in the forthcoming (eighth) edition of the TNM classification for lung cancer. $J$ Thorac Oncol 2016;11:39-51.

[6] Vansteenkiste J, Crinò L, Dooms C, Douillard JY, Faivre-Finn C, Lim E, et al. 2nd ESMO consensus conference on lung cancer: earlystage non-small-cell lung cancer consensus on diagnosis, treatment and follow-up. Ann Oncol 2014;25:1462-1474.

[7] Travis WD. Pathology of lung cancer. Clin Chest Med 2011;32:669692.

[8] Cancer Genome Atlas Research Network. Comprehensive molecular profiling of lung adenocarcinoma. Nature 2014;511:543-550.

[9] Cancer Genome Atlas Research Network. Comprehensive genomic characterization of squamous cell lung cancers. Nature 2012;489:519-525.

[10] Tsao AS, Scagliotti GV, Bunn PA Jr, Carbone DP, Warren GW, Bai C, et al. Scientific advances in lung cancer 2015. J Thorac Oncol 2016;11:613-638.

[11] Broët P, Camilleri-Broët S, Zhang S, Alifano M, Bangarusamy D, Battistella M, et al. Prediction of clinical outcome in multiple lung cancer cohorts by integrative genomics: implications for chemotherapy selection. Cancer Res 2009;69:1055-1062.

[12] Giangreco A, Groot KR, Janes SM. Lung cancer and lung stem cells: strange bedfellows? Am J Respir Crit Care Med 2007;175:547-553.

[13] Pikor LA, Ramnarine VR, Lam S, Lam WL. Genetic alterations defining NSCLC subtypes and their therapeutic implications. Lung Cancer 2013;82:179-189.

[14] Sato M, Shames DS, Gazdar AF, Minna JD. A translational view of the molecular pathogenesis of lung cancer. J Thorac Oncol 2007;2:327-343.

[15] Sandler A, Gray R, Perry MC, Brahmer J, Schiller JH, Dowlati A, et al. Paclitaxel-carboplatin alone or with bevacizumab for non-smallcell lung cancer. $N$ Engl J Med 2006;355:2542-2550.

[16] Thatcher N, Hirsch FR, Luft AV, Szczesna A, Ciuleanu TE, Dediu $\mathrm{M}$, Ramlau R, et al. Necitumumab plus gemcitabine and cisplatin versus gemcitabine and cisplatin alone as first-line therapy in patients with stage IV squamous non-small-cell lung cancer (SQUIRE): an open-label, randomised, controlled phase 3 trial. Lancet Oncol 2015;16:763-774.

[17] Hata A, Katakami N, Yoshioka H. How sensitive are epidermal growth factor receptor-tyrosine kinase inhibitors for squamous cell carcinoma of the lung harboring EGFR gene-sensitive mutations? $J$ Thorac Oncol 2014;9:e20.

[18] Győrffy B, Surowiak P, Budczies J, Lánczky A. Online survival analysis software to assess the prognostic value of biomarkers using transcriptomic data in non-small-cell lung cancer. PLoS One 2013;8:e82241.

[19] Balaj L, Lessard R, Dai L, Cho YJ, Pomeroy SL, Breakefield XO, et al. Tumour microvesicles contain retrotransposon elements and amplified oncogene sequences. Nat Commun 2011;2:180.

[20] Milane L, Singh A, Mattheolabakis G, Suresh M, Amiji MM. Exosome mediated communication within the tumor microenvironment. J Control Release 2015;219:278-294.

[21] Sharma S, Zuñiga F, Rice GE, Perrin LC, Hooper JD, Salomon C. Tumor-derived exosomes in ovarian cancer - liquid biopsies for early detection and real-time monitoring of cancer progression. Oncotarget 2017;8:104687-104703.

[22] Gettins PG. Serpin structure, mechanism, and function. Chem Rev 2002;102:4751-4804.

[23] Chmelař J, Kotál J, Langhansová H, Kotsyfakis M. Protease inhibitors in tick saliva: the role of serpins and cystatins in tick-hostpathogen interaction. Front Cell Infect Microbiol 2017;7:216.

[24] Rau JC, Beaulieu LM, Huntington JA, Church FC. Serpins in thrombosis, hemostasis and fibrinolysis. J Thromb Haemost. 2007;5 Suppl 1:102-115.

[25] Irving JA, Ekeowa UI, Belorgey D, Haq I, Gooptu B, Miranda E, et al. The serpinopathies studying serpin polymerization in vivo. Methods Enzymol 2011;501:421-466.

[26] Silverman GA, Bird PI, Carrell RW, Church FC, Coughlin PB, Gettins PG, et al. The serpins are an expanding superfamily of structurally similar but functionally diverse proteins. Evolution, mechanism of inhibition, novel functions, and a revised nomenclature. J Biol Chem 2001;276:33293-6.

[27] Carrell RW, Read RJ. How serpins transport hormones and regulate their release. Semin Cell Dev Biol 2017;62:133-141.

[28] Gooptu B, Dickens JA, Lomas DA. The molecular and cellular pathology of $\alpha \square$-antitrypsin deficiency. Trends Mol Med 2014;20:116-127.

[29] Mazzoccoli G, Pazienza V, Panza A, Valvano MR, Benegiamo G, Vinciguerra M, et al. ARNTL2 and SERPINE1: potential biomarkers for tumor aggressiveness in colorectal cancer. J Cancer Res Clin Oncol 2012;138:501-511.

[30] Huber MA, Kraut N, Beug H. Molecular requirements for epithelialmesenchymal transition during tumor progression. Curr Opin Cell Biol 2005; 17:548-558.

[31] Peinado H, Olmeda D, Cano A. Snail, Zeb and bHLH factors in tumour progression: an alliance against the epithelial phenotype? Nat Rev Cancer 2007;7:415-428.

[32] Acloque H, Adams MS, Fishwick K, Bronner-Fraser M, Nieto MA Epithelial-mesenchymal transitions: the importance of changing cell state in development and disease. J Clin Invest. 2009;119:1438-1449.

[33] Thiery JP, Acloque H, Huang RY, Nieto MA. Epithelial-mesenchymal transitions in development and disease. Cell 2009;139:871-890.

[34] Chaffer CL, Weinberg RA. A perspective on cancer cell metastasis. Science 2011;331:1559-1564.

[35] Yang J, Weinberg RA. Epithelial-mesenchymal transition: at the crossroads of development and tumor metastasis. Dev Cell 
2008;14:818-829.

[36] Mansfield KJ, Hughes JR. P2Y receptor modulation of ATP release in the urothelium. Biomed Res Int 2014;2014:830374.

[37] Nishi H, Arai H, Momiyama T. NCI-H295R, a human adrenal cortexderived cell line, expresses purinergic receptors linked to $\mathrm{Ca}^{2+}$ mobilization/influx and cortisol secretion. PLoS One 2013;8:e71022.

[38] Yi X, Zhou Q, Wang C, Lin J, Liu P, Fu C. Platelet receptor Gene (P2Y12, P2Y1) and platelet glycoprotein Gene (GPIIIa) polymorphisms are associated with antiplatelet drug responsiveness and clinical outcomes after acute minor ischemic stroke. Eur J Clin Pharmacol 2017;73:437-443.

[39] Tan Y, Zhang T, Zhou L, Liu S, Liang C. MiR-34b-3p represses the multidrug-chemoresistance of bladder cancer cells by regulating the CCND2 and P2RY1 genes. Med Sci Monit 2019;25:1323-1335.

[40] Kim IW, Han N, Kim MG, Kim T, Oh JM. Copy number variability analysis of pharmacogenes in patients with lymphoma, leukemia, hepatocellular, and lung carcinoma using the cancer genome atlas data. Pharmacogenet Genomics 2015;25:1-7.

[41] Shah N, Khurana S, Cheng K, Raufman JP. Muscarinic receptors and ligands in cancer. Am J Physiol Cell Physiol 2009;296:C221-32.

[42] Lin G, Sun L, Wang R, Guo Y, Xie C. Overexpression of muscarinic receptor 3 promotes metastasis and predicts poor prognosis in nonsmall-cell lung cancer. J Thorac Oncol 2014;9:170-178.

[43] Larue L, Bellacosa A. Epithelial-mesenchymal transition in development and cancer: role of phosphatidylinositol 3' kinase/AKT pathways. Oncogene 2005;24:7443-54.

[44] Wang Y, Li J, Wen S, Yang X, Zhang Y, Wang Z, et al. CHRM3 is a novel prognostic factor of poor prognosis in patients with endometrial carcinoma. Am J Transl Res 2015;7:902-911.

[45] Rani S, Corcoran C, Shiels L, Germano S, Breslin S, Madden S, et al. Neuromedin U: a candidate biomarker and therapeutic target to predict and overcome resistance to HER-tyrosine kinase inhibitors. Cancer Res 2014;74:3821-33.

[46] Wu Y, McRoberts K, Berr SS, Frierson HF Jr, Conaway M, Theodorescu D. Neuromedin $U$ is regulated by the metastasis suppressor RhoGDI2 and is a novel promoter of tumor formation, lung metastasis and cancer cachexia. Oncogene 2007;26:765-773.

[47] You S, Gao L. Identification of NMU as a potential gene conferring alectinib resistance in non-small cell lung cancer based on bioinformatics analyses. Gene 2018;678:137-142.

[48] Brindley DN. Lipid phosphate phosphatases and related proteins: signaling functions in development, cell division, and cancer. $J$ Cell Biochem 2004;92:900-912.

[49] Fang X, Gaudette D, Furui T, Mao M, Estrella V, Eder A, et al. Lysophospholipid growth factors in the initiation, progression, metastases, and management of ovarian cancer. Ann N Y Acad Sci 2000;905:188-208.

[50] Shida D, Watanabe T, Aoki J, Hama K, Kitayama J, Sonoda H, et al. Aberrant expression of lysophosphatidic acid (LPA) receptors in human colorectal cancer. Lab Invest 2004;84:1352-1362.

[51] Hayashi M, Okabe K, Yamawaki Y, Teranishi M, Honoki K, Mori $\mathrm{T}$, et al. Loss of lysophosphatidic acid receptor-3 enhances cell migration in rat lung tumor cells. Biochem Biophys Res Commun 2011;405:450-454.

[52] $\mathrm{Hu}$ WM, Li L, Jing BQ, Zhao YS, Wang CL, Feng L, et al. Effect of S1P5 on proliferation and migration of human esophageal cancer cells. World J Gastroenterol 2010;16:1859-1866.

[53] Novgorodov AS, El-Alwani M, Bielawski J, Obeid LM, Gudz TI. Activation of sphingosine-1-phosphate receptor S1P5 inhibits oligodendrocyte progenitor migration. FASEB J 2007;21:1503-1514.

[54] Balthasar S, Samulin J, Ahlgren H, Bergelin N, Lundqvist M, Toescu EC, et al. Sphingosine 1-phosphate receptor expression profile and regulation of migration in human thyroid cancer cells. Biochem $J$ 2006;398:547-556.

[55] Huang YL, Chang CL, Tang CH, Lin YC, Ju TK, Huang WP, et al. Extrinsic sphingosine 1-phosphate activates S1P5 and induces autophagy through generating endoplasmic reticulum stress in human prostate cancer PC-3 cells. Cell Signal 2014;26:611-618.

[56] Chang CL, Ho MC, Lee PH, Hsu CY, Huang WP, Lee H. S1P(5) is required for sphingosine 1-phosphate-induced autophagy in human prostate cancer PC-3 cells. Am J Physiol Cell Physiol 2009;297:C451-458. 